\title{
The ORNATE India Project: United Kingdom-India Research Collaboration to tackle visual impairment due to diabetic retinopathy
}

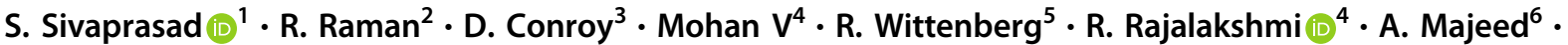 \\ S. Krishnakumar ${ }^{2} \cdot$ T. Prevost ${ }^{6} \cdot$ S. Parameswaran ${ }^{2} \cdot$ P. Turowski $^{3} \cdot$ U. Maheswari $^{7} \cdot$ R. Khobragade $^{8} \cdot$ G. Netuveli $^{9}$. \\ R. Sadanandan ${ }^{8} \cdot$ J. Greenwood $\mathbb{D}^{3} \cdot \mathrm{K}$. Ramasamy ${ }^{10} \cdot \mathrm{M}$. Rao ${ }^{6} \cdot$ C. Bergeles ${ }^{11} \cdot$ T. Das $\mathbb{D}^{12} \cdot$ for the ORNATE India \\ Project Group
}

Received: 28 February 2020 / Revised: 15 March 2020 / Accepted: 17 March 2020 / Published online: 12 May 2020

(c) The Author(s), under exclusive licence to The Royal College of Ophthalmologists 2020

\begin{abstract}
Introduction The ORNATE India project is funded by the UK Research and Innovation (UKRI) through the Global Challenges Research Fund. The aim is to build research capacity and capability in India and the UK to tackle global burden of diabetes-related visual impairment. As there are over 77 million people with diabetes in India, it is challenging to screen every person with diabetes annually for sight-threatening diabetic retinopathy (DR). Therefore, alternate safe approaches need to be developed so that those at-risk of visual impairment due to DR is identified promptly and treated.

Methods The project team utilised diverse global health strategies and research methods to co-design work packages to build research capacity and capability to ensure effective, affordable and efficient DR services are made available for the population. The strategies and methods employed included health system strengthening; implementation science; establishing care pathways; co-designing collaborative studies on affordable technologies, developing quality standards and guidelines to decrease variations in care; economic analysis; risk modelling and stratification. Five integrated work packages have been developed to deal with all aspects of DR care. These included implementation of a DR screening programme in the public health system in a district in Kerala, evaluating regional prevalence of diabetes and DR and assessing ideal tests for holistic screening for diabetes and its complications in 20 areas in India, utilising artificial intelligence on retinal images to facilitate DR screening, exploring biomarker and biosensor research to detect people at risk of diabetes complications, estimating cost of blindness in India and risk modelling to develop risk-based screening models for diabetes and its complications. A large collaborative network will be formed to propagate research, promote shared learning and bilateral exchanges between high- and middle-income countries to tackle diabetes-related blindness.
\end{abstract}

Members of the ORNATE India Project Group are listed below Acknowledgements.

S. Sivaprasad

senswathi@aol.com

1 NIHR Biomedical Research Centre, Moorfields Eye Hospital NHS Foundation Trust, London, UK

2 Vision Research Foundation, Sankara Nethralaya, Chennai, India

3 UCL Institute of Ophthalmology, London, UK

4 Madras Diabetes Research Foundation, Chennai, India

5 University of Oxford, Oxford, UK
6 Imperial College London, London, UK

SASTRA Deemed University, Chennai, India

8 Government of Kerala, Thiruvanathapuram, India

University of East London, London, UK

10 Aravind Medical Research Foundation, Madurai, India

11 King's College London, London, UK

12 Hyderabad Eye Research Foundation, L V Prasad Eye Institute, Hyderabad, India 


\section{Introduction}

\section{Global health challenge}

All countries are committed to the United Nations 2030 Sustainable Development Goals (SDG) and have aligned their national priorities to accomplish their implementation. However, low- and middle-income countries (LMIC) are performing below expectations, especially on health and well-being (SDG 3). This directly impacts economic growth (SDG 8), education (SDG 4) and gender equity (SDG 5) [1]. The Global Challenges Research Fund (GCRF) was set up by the UK Government in 2015 and is administered through its delivery partners, for example, United Kingdom Research and Innovation (UKRI), to address complex global development challenges and support collaborative research that will improve the economic prosperity, welfare and quality of life of people in LMIC. The ORNATE India project, funded by GCRF and UKRI, is a UK-India research collaboration aimed at building research capacity and capability in the UK and India to tackle the burden of diabetes-related visual impairment.

Diabetes is a worldwide epidemic. Nearly $80 \%$ of adults with diabetes reside in LMIC where primary care infrastructure is in its infancy, fuelling the global challenge of managing diabetes and its complications [2]. Undiagnosed diabetes is a significant problem in these countries and a high proportion of patients are only diagnosed when they present with a disease-related complication. For example, India is a home to a large number of people with diabetes with significant regional variations in the prevalence of the disease [2, 3]. It is estimated that in 2019, there were 77 million adults (20-79 years) with diabetes in India and this number is predicted to increase to 100.95 million in 2030 and 134.23 million in 2045 [4]. The age-adjusted prevalence of diabetes in India is projected to increase from current $10.4 \%$ to $11.2 \%$ in year 2030 and to $11.5 \%$ in year 2045. In addition, an estimated further 43.9 million people have undiagnosed diabetes (Fig. 1) [4].

Laboratory tests for diabetes are costly, and not readily available in primary care services in LMIC, the estimated prevalence of diabetes is in excess of the capacity of public health initiatives to screen the population, persons diagnosed with diabetes are not regularly monitored and majority of the population are not aware of diabetes and its complications. Therefore, there is an unmet need to find reliable and affordable ways to identify the population at risk of diabetes so that these persons can then undergo the definitive diagnostic tests for diabetes [5, 6].

In addition, there is no systematic screening for the complications of the disease. Implementation of screening programmes for complications for all people with diabetes is not economically or logistically viable in resourceconstrained countries especially in India due to the sheer numbers of people with diabetes. So, small steps need to be taken to initiate targeted screening of the population most at-risk of complications of diabetes. One approach is to develop models and risk scores with minimal variables that can be applied to LMIC where data are limited.

Another step is to initiate risk-based screening pathways to identify and ensure early management of all diabetes complications in people most at-risk of complications based on the fact that microvascular and macrovascular disease have strong associations. For example, identifying and
Fig. 1 This infographics on the burden of diabetes in India is developed from the International Diabetes Federation Atlas highlighting the expected near doubling of prevalence of diabetes from 2019 to 2045 .

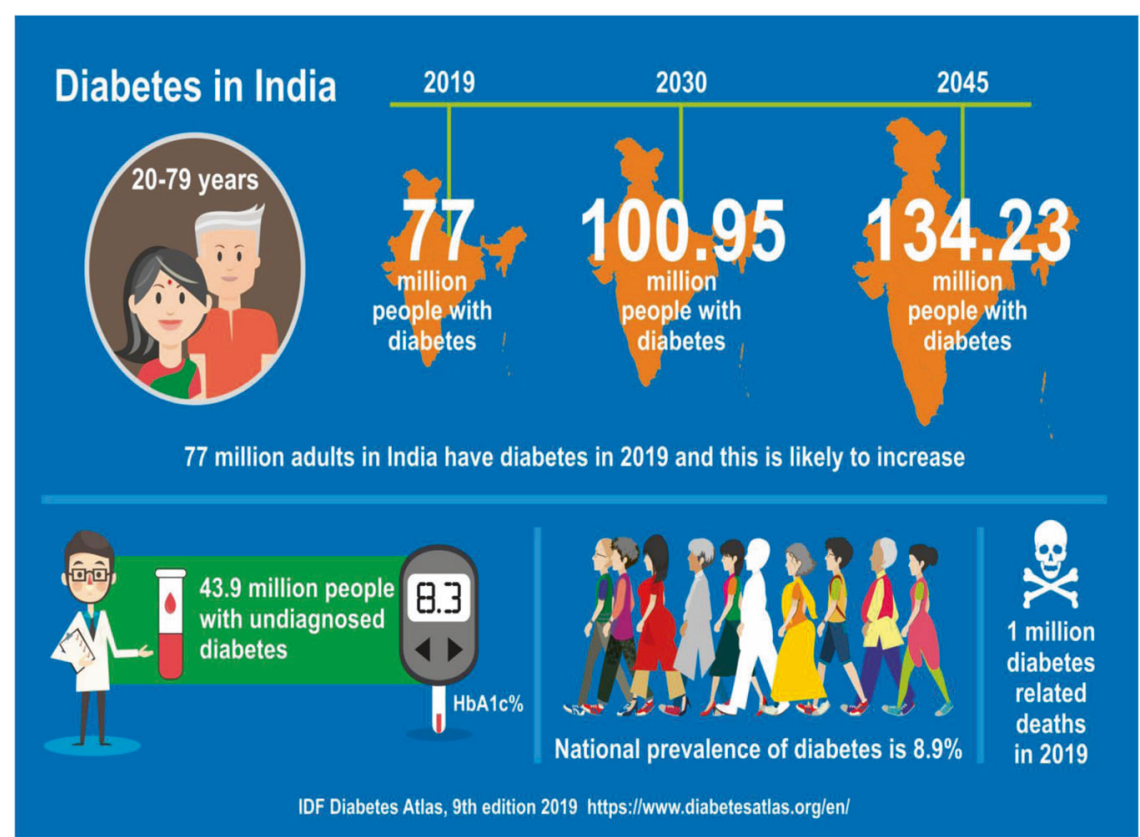


treating those most at risk of sight threatening retinopathy will not only reduce rates of visual impairment but also identify the group that needs screening for other complications. This approach is most applicable in LMIC, where several barriers hinder the set up of a systematic screening programme for the whole population with diabetes.

\section{The global challenge of visual impairment due to sight threatening diabetic retinopathy}

Globally, an estimated 36 million people were blind in 2015 and of these 8.8 million lived in India. In addition, another 47.7 million in India had moderate and severe visual impairment [7]. Uncorrected refractive error and cataract are the most common causes of visual impairment and blindness, respectively. However, due to relentlessly increasing prevalence of type 2 diabetes in India, diabetic retinopathy (DR) is emerging as a leading cause of vision impairment in working adults in urban India and is predicted to increase from 4.21 million in 2020 to 6.08 million by year 2030 [8]. Early detection and prompt treatment will enable prevention of diabetes-related visual impairment. As in most LMIC, there are no systematic national or state-wide screening programmes for DR in India. Retinal examinations or retinal photography are performed opportunistically when people with diabetes visit an eye facility, though often only after vision loss [9].

The two most common causes of visual impairment due to sight-threatening diabetic retinopathy (STDR) are diabetic macular oedema (DMO) and complications caused by proliferative diabetic retinopathy (PDR). Both conditions are treatable; but the patients may remain asymptomatic for a considerable time before manifesting with moderate to severe visual impairment. Therefore, screening utilising retinal cameras or retinal examination by trained ophthalmologists or optometrists is crucial for timely referral for treatment to prevent vision loss. In addition to the numbers of challenges of higher disease burden, low resources and inadequate trained workforce, there is also a need to develop management guidelines that are relevant to local needs and resources to standardise DR care in LMIC.

Compared with high-income countries, there are inadequate resources to purchase costly standard retinal cameras in LMIC. Secondly, laser photocoagulation remains the treatment of choice in LMIC for both DMO and PDR as repeated intravitreal injections of anti-vascular endothelial growth factor (anti-VEGF) agents for DMO are too costly. In fact, laser devices are also costly and many people in LMIC have no access to either of these treatments. The more complex complications of PDR need to be managed by trained vitreo-retinal surgeons and require significant infrastructure and these are rarely available in LMIC either.
In order to provide access to a DR care pathway to all who need it and reduce health inequality, there is an urgent need to upskill the health workforce in India, as well as, train additional health professionals, strengthen data quality, governance and accountability of care models to ensure sustainability and scalability of good practice. A solution to the shortage of ophthalmologists is to urgently train and prepare allied specialities to cross their current boundaries to acquire new strengths, skills and competencies through research, practice, policy and education to diminish the gap between demand to clinical services and workforce deficiency [10].

\section{Shared learning and exchanges from the UK}

The UK is one of the high-income countries where diabetes care pathways have been well established for decades with well-equipped primary care infrastructure to cater for about four million people with diabetes and its complications. The National Health Service Eye Screening Programme was established in 2003 [11]. People identified from the screening programmes are referred for treatment to secondary care where they are treated using retinal laser and/or anti-VEGF [12]. The treatment is free of cost to patients at their point of access and care is delivered to high-quality standards. At least $80 \%$ of people with diabetes in the UK are recalled for annual screening for vision threatening complications. These systematic approaches in the UK have resulted in DR being no longer the most common cause of blindness in the working age group [13]. There is an unmet need to share good practices from the UK and co-develop new ways of tackling diabetes-related visual impairment with other countries.

In addition, as the NHS also has finite resources, reducing overall costs of existing programmes through innovation remains a key aim of all research [14].

A screening episode costs approximately $£ 35$ and so for the current population of four million people with diabetes, the national annual health expenditure is about $£ 140$ million. Therefore, innovative approaches to screening for STDR will also be beneficial in high-income countries. There are no such published data on costs for DR care from India.

As DR can only be diagnosed by a retinal examination, it is a formidable task to screen 77 million people with diabetes in India based on the UK screening programme. Alternative options need to be explored. Recent advances in this area include the use of artificial intelligence (AI) to grade retinal images, progression of tele-ophthalmology programmes and the availability of less expensive retinal cameras $[15,16]$. Despite development and validation of these methods of retinal screening, additional responsibility 
lies in identifying people with treatable DR and those at high risk of developing other end organ damage.

Identifying a panel of biomarkers that may be useful to screen for complications of diabetes may appear a futuristic but an avenue worth exploring. In order to have enhanced value, the tests must be applicable for any nation to adopt and adapt and so the models should be underpinned by a robust health economic evaluation to support decisionmaking processes in local settings. These are, therefore, opportunities for collaborative research between organisations in high-income countries and LMIC to share good practices and develop new ways of tackling diabetes-related visual impairment, which would be of benefit globally.

\section{Aims of the ORNATE India project}

The ORNATE India project is a multidisciplinary, multifaceted UK-India collaborative study funded by the GCRF and UKRI. The project team consists of ophthalmologists, diabetologists, public health experts, primary care physicians, research scientists, biostatisticians, computer engineers, sensor technology experts, health economists, data quality assurance and project managers. The aim of this multidisciplinary team is to contribute to the targets of the SDGs in terms of improving good health and well-being, promoting economic development and welfare, improving education and decreasing health inequality by tackling the global challenge of visual impairment in people with diabetes. By increasing research capacity and capability, the ORNATE India project will answer the following ten questions:

(1) Can a clinically and cost-effective DR care pathway using smartphone retinal cameras and telemedicine, be initiated in a public health system in India?

(2) What is the regional prevalence of DR and vision impairment in people with diabetes in India?

(3) Can a cost-effective multistate telemedicine programme, incorporating a house-to-house survey, be employed in India to screen for diabetes and its complications?

(4) How can AI be used for detecting DR and other complications?

(5) What is the agreement of point-of-care kits and nonlaboratory tests in the diagnosis of diabetes?

(6) Can systemic biomarkers be used accurately to identify risks of complications in diabetics?

(7) Can point-of-care blood sensors be developed to aid diagnosis of diabetes and its complications?

(8) Can predictive models for diabetes complications be developed that are appropriate for LMIC?

(9) Can the formation of a DR Clinical Network in India help to guide clinical practice?
(10) What aspects of the findings of this project can be translated globally or can be used to model reverse innovation of DR screening in the UK?

\section{Methods}

In order to answer these research questions by increasing research capacity and capability, the project designed work packages utilising diverse global health strategies and research methods. As the public health system is under developed and the majority of the low socio-economic strata of the population are dependent on these services, one crucial step was to strengthen the health system through research. Theories underpinning implementation science were considered to ensure that clinical research findings could be systematically absorbed for scale up and sustaining universal health coverage. Another challenge is the variations of DR care in India mainly driven by the cost of treatment of sight threatening DR. Therefore, collaborative studies had to be designed to develop clinical standards and guidelines that are applicable to the local environment.

The research questions above were addressed in five interlinked work packages summarised in Table 1.

\section{Work Package 1a: Integration of DR care pathway in the public health system}

The Nayanamritham study will investigate the clinical efficacy and cost-effectiveness of initiating a DR care pathway from screening to treatment in the public health system of Kerala spanning primary, secondary and tertiary care. A pilot study will be undertaken across 16 family health centres (FHCs) in Thiruvananthapuram district of Kerala in collaboration with the Government of Kerala. The components of the project will include capacity and capability strengthening at the FHCs and secondary care. This study will evaluate the prevalence of DR and referable DR among the persons who have already been diagnosed with diabetes who attend primary care settings. The process evaluation of this DR care pathway and economic evaluation of the newly developed service will be reported.

\section{1b: Multistate telemedicine project}

The SMART India study is a 20-centre cross-sectional study on adults aged 40 years or above residing in homes in predefined urban and rural clusters across twelve States and one Union Territory in India. Trained field workers will conduct house-to-house health surveys to collect data on demography, social, economic, anthropometry, quality of life and life satisfaction. In addition, point-of-care blood tests will be done. 
Table 1 Mapping of research aims to project epistemology and architecture.

\begin{tabular}{l} 
Research questions \\
\hline 1. Can a clinically and cost-effective DR \\
care pathway using smartphone cameras \\
and telemedicine be initiated in a public \\
health system in India?
\end{tabular}

health system in India?

2. What is the regional prevalence of diabetic retinopathy (DR) and vision impairment in people with diabetes in India?

3. Can a cost-effective multistate telemedicine programme incorporating a house-to-house survey be employed in India to screen for diabetes and its complications?

4. What is the diagnostic accuracy of pointof-care kits and non-laboratory tests for screening for diabetes?

5. How can we use artificial intelligence (AI) to detect DR and other complications? programmes exist in India.

6. Can systemic biomarkers be used to accurately identify people with diabetes at risk of diabetic retinopathy and can a biosensor be developed to detect candidate biomarkers in blood?

8. Can we develop predictive models for diabetes complications that can be applied to $\mathrm{LMIC}$ ? DR are available in small studies.

9. Can we develop a national diabetic retinopathy research network in India that can help guide clinical practices there? 10. What aspects of the findings of this project can be translated globally or can be used to model reverse innovation of DR screening in the UK?
Several reports on use of AI have shown

Multiple local tele-ophthalmology

No published data on DR scr
public health system in India.

Epidemiological data on DR exist mainly in The regional prevalence of DR and visual 1 South India.

Random blood sugar is the most commo test that is used to screen for diabetes.

excellent diagnostic accuracy of AI compared with ground truth.

Multiple reports on circulating markers in

Currently, screening for the many different complications of diabetes is done in separate specialist clinics

There are no national ophthalmology research networks in India.

The NHS Eye Screening Programme is a

\begin{tabular}{ll} 
What will the project add & $\begin{array}{l}\text { Work } \\
\text { packages }\end{array}$ \\
\hline
\end{tabular}

1,4 people with diabetes registered in the noncommunicable disease registers in the public system in Thiruvananthapuram district in Kerala to report process evaluation, clinical and cost-effectiveness of the care pathway to enable scalability and sustainability and translation to other regions in India. impairment in people with diabetes in predefined rural and urban clusters in 12 regions in India. This will inform whether DR screening should be a priority in all states in India.

Evaluate whether a holistic diabetes 1,4 complications telemedicine project can be introduced in India using a minimal dataset.

Agreement of point-of-care $\mathrm{HbA} 1 \mathrm{c}$ and 1,5 other non-laboratory tests will be reported to identify diabetes screening tools that can be accurately applied in LMIC where laboratory tests are not always available.

Quality assessment of retinal images captured with low-cost non-mydriatic retinal cameras and development and validation of AI on these images.

Diagnostic accuracy of 12 circulating biomarkers will be done to identify a panel of markers that may be used in a biosensor to detect sight threatening DR.

By creating predictive tools, all clinical 5 specialists will be able to predict the risk of developing other complications of diabetes and ensure timely referral.

Development of a national diabetic 5 retinopathy research network will enable joint efforts in developing clinical standards appropriate to India using data from retrospective and longitudinal studies.

(s) are models using 5 werlistished DR care pathway but there outputs from various work packages such are finite resources. Many LMIC do not as AI incorporated into low cost cameras, have systematic DR screening pathways. predictive models and biosensors.
Retinal images will be captured at sites using hand-held nonmydriatic cameras and uploaded to a web-based portal to the local research centre for primary grading and to four tertiary centres for secondary grading. A retinal specialist in the four tertiary centres will arbitrate any discrepancy between primary and secondary grading results. The results are entered into the web-based database and the local centre disseminates the results to the patients and arranges onward referral and treatment, if required. This study will also evaluate the agreement of laboratory and non-laboratory-based tests in diagnosing diabetes and its complications, the clinical and cost-effectiveness of running a multistate telemedicine programme for DR, report any regional variations in the prevalence of diabetes and DR, and conduct a process evaluation on the telemedicine programme utilising non-mydriatic retinal cameras. A detailed study protocol will be published. 
Work Package $1 \mathrm{~b}$ : Integration of DR care pathway in the public health system

The Nayanamritham study will investigate the clinical efficacy and cost-effectiveness of initiating a DR care pathway from screening to treatment in the public health system of Kerala spanning primary, secondary and tertiary care. A pilot study will be undertaken across 16 FHCs in Thiruvananthapuram district of Kerala in collaboration with the Government of Kerala. The components of the project will include capacity and capability strengthening at the FHCs and secondary care. This study will evaluate the prevalence of DR and referable DR among the persons who have already been diagnosed with diabetes who attend primary care settings. The process evaluation of this DR care pathway and economic evaluation of the newly developed service will be reported.

\section{Work Package 2: Automated tools in the detection of DR}

This is a technological collaboration where we aim to develop and/or validate automated tools using AI to grade for DR manifestations and develop novel uses of AI of the retina. We also aim to develop or validate image quality assessment tools to be incorporated into the cameras used in Work Package 1 to assist the allied health workers to capture gradable images. The work package will also seek collaboration to scope out an ideal retinal camera integrated with OCT.

\section{Work Package 3: Novel diagnostic technologies}

This is a laboratory-based collaboration between the UK and India to investigate if circulating biomarkers can be used to identify STDR. This prospective multicentre case control study will be conducted in parallel in the UK and India for which we aim to recruit a total of 500 participants. The study protocol and statistical analysis plan will detail the methods for the selection of candidate biomarkers and a recommended cross-validation approach will be used to detect effective combinations for the principal study outcomes of STDR. This work package will also involve the development of a blood biosensor in India to test $\mathrm{HbAlc}$ and any other circulating biomarkers if found to be predictive for STDR in the above biomarker study.

\section{Work Package 4: Economic analysis}

This work package will provide the health economics input to the Smart India and Nayanamritham studies in Work Package 1 above as well as an estimate of the cost of blindness in India. The objectives of the economic analyses are to estimate the costs, cost consequences and costeffectiveness of the screening and care pathway in the Kerala pilot programme and in the SMART India programme. Our cost estimates include the costs of staff training and equipment (suitably annuitised), the costs of staff time on screening, grading images and providing treatment, the costs of travel by the patient and accompanying family member or accredited social health activist (ASHA) workers and opportunity costs of time for the family members or ASHA workers. For the cost consequences analyses we will treat as the comparator no screening and no baseline treatment, leading to severe visual impairment or blindness. The outcomes will therefore be estimated as severe visual impairment or blindness averted as a result of screening and subsequent treatment. For the cost-effectiveness analyses we will use evidence from past studies on the number of quality-adjusted life years lost by a person who experiences severe visual impairment or blindness.

\section{Work Package 5a: Risk modelling}

In this work package, we will use multiple databases from primary and secondary care and previous epidemiological studies from diverse ethnic cohorts to develop, validate and calibrate risk models on various aspects of diabetes and its complications so that they can be applied to LMIC. In particular, the relation between the complications of diabetes, triage tools to risk stratify the diabetes population that requires urgent attention, and novel care pathways will be evaluated.

\section{Work Package 5b: Collaborative research network in India}

In Addition, in India, we will work on retrospective data from routine clinical practice and conduct a Delphi exercise to evaluate the current clinical practice in the management of various aspects of DR and a consensus statement on recommended clinical standards will be developed that are applicable to LMIC. This is particularly important as reports on the outcomes of STDR management in LMIC are sparse and current pathways in high-income countries such as the UK are not translatable to LMIC.

\section{Pathways to impact and legacy}

This collaboration between the UK and India is likely to have significant global impact as the outcomes of the ORNATE India project could be applied to LMIC with far reaching potential to promote health and economic benefits. The outputs from the work packages will facilitate the goal of universal access to equitable, affordable and quality DR screening that is practical in all LMIC (Table 2). 
Table 2 Planned interactions and lessons to be shared.

Integrating research into clinical practice

Improved governance of research projects from inception of research idea to dissemination of output

Improved data capture and quality in all work packages

Importance of data protection

Development of protocols and protocol guided screening and treatment pathways

Creation of statistical analysis plans for studies

Co-development of biomarker lab standard operating procedures

Increased awareness of the need for economic evaluation of services and interventions

Local capacity and capability building to decrease global health inequity

Establishment of robust network of researchers

Need for infrastructure development to be research friendly

Strengthening public health system for diabetic retinopathy screening

Research can be used to contribute to sustainable development goals Improved awareness that lessons from low income countries can be used to reverse innovate high-income countries

In the short-term, the project could help to design guidelines and policy for DR screening and treatment pathways for STDR that are applicable to LMIC.

We expect that the development of an affordable biosensor will empower people with diabetes to self-monitor their health.

New knowledge and hands-on experience obtained by skilled and unskilled workers, improved capacity in different types of healthcare institutions, public engagement, advocacies and public awareness of DR through our research programme in different regions of India will also improve the uptake of screening programmes and treatment and thus reduce visual impairment in the long run. The increased research capability will lead to effective change of existing organisational cultures and research policies where needed.

Increased research capability in the UK will result in more research outputs in this field and the creation of additional international multidisciplinary teams during and after the lifetime of the grant.

\section{Conclusion}

In conclusion, the ORNATE India study, funded by GCRF and UKRI, aims to develop sustainable healthcare for people with diabetes to decrease visual impairment. Codesigned innovative approaches planned in this project are focused on increase capacity and capability in LMIC, so that in the long-term, health inequality will be reduced, and diabetes and blindness-related poverty alleviated, and productivity improved.

\section{Summary}

\section{What was known before}

- The United Kingdom has one of the most effective DR screening programmes in the world. Systematic screening and timely treatment have resulted in sight threatening DR to be no longer the most common cause of blindness in the working age group in the UK. LMIC have constrained resources to replicate the UK screening programme despite a surge in numbers of people with diabetes.

\section{What this study adds}

- This UK-India project aims to tackle visual impairment due to DR by increasing research capacity and capability in multiple studies. These include implementation of a DR screening programme in the public health system in a district in Kerala, evaluating tests that could be used for holistic screening for diabetes and its complications in 20 areas in India, utilising AI on retinal images to facilitate DR screening, exploring biomarker and biosensor research to detect people at risk of diabetes complications, estimating cost of blindness in India and risk modelling to develop risk-based screening models for diabetes and its complications in LMIC. A large collaborative network will also be developed in India to boost research.

Acknowledgements The ORNATE India project is funded by the GCRF UKRI (MR/P207881/1). The research is supported by the NIHR Biomedical Research Centre at Moorfields Eye Hospital NHS Foundation Trust and UCL Institute of Ophthalmology.

The ORNATE India Project Group Pramod Bhende ${ }^{13}$, Padmaja K. Rani ${ }^{14}$, Rupak Roy ${ }^{15}$, Supita Das ${ }^{15}$, V. Narendran ${ }^{16}$, George Manayath $^{16}$, Giridhar Anantharaman ${ }^{17}$, Mahesh Gopalakrishnan ${ }^{17}$, Sundaram Natarajan ${ }^{18}$, Radhika Krishnan ${ }^{18}$, Sheena Liz Mani ${ }^{19}$, Manisha Agarwal $^{20}$, Tapas Padhi ${ }^{21}$, Umesh Behera ${ }^{21}$, Harsha Bhattacharjee ${ }^{22}$, Manabjyoti Barman ${ }^{22}$, Gajendra Chawla ${ }^{23}$, Alok Sen ${ }^{24}$, Moneesh Saxena $^{25}$, Asim K. Sil ${ }^{26}$, Subhratanu Chakabarty ${ }^{26}$, Thomas Cherian ${ }^{27}$, Reesha $\mathrm{KR}^{27}$, Rushikesh Naigaonkar ${ }^{28}$, Abishek Desai ${ }^{28}$, Col Madan Deshpande $^{29}$, Sucheta Kulkarni ${ }^{29}$

${ }^{13}$ Sankara Nethralaya, Chennai, Tamil Nadu, India; ${ }^{14}$ LV Prasad Eye Institute, Hyderabad, Telangana, India; ${ }^{15}$ Sankara Nethralaya, Kolkata, India; ${ }^{16}$ Aravind Eye Hospital, Coimbatore, Tamil Nadu, India; ${ }^{17}$ Giridhar Eye Institute, Cochin, Kerala, India; ${ }^{18}$ Aditya Jyot Hospital, Mumbai, Maharashtra, India; ${ }^{19} \mathrm{Dr}$ Tony Fernandez Eye 
Hospital, Aluva, Kerala, India; ${ }^{20}$ Dr Shroff's Charity Eye Hospital, New Delhi, India; ${ }^{21}$ LV Prasad Eye Institute, Bhubaneshwar, Odisha, India; ${ }^{22}$ Sri Sankaradeva Nethralaya, Guwahati, Assam, India; ${ }^{23}$ Vision Academy- The Socio Medical Society, Bhopal, Madhya Pradesh, India; ${ }^{24}$ Sadguru Netra Chikitsalaya, Chitrakoot, Madhya Pradesh, India; ${ }^{25}$ Aurobindo Nethralaya, Raipur, Chhattisgarh, India; ${ }^{26}$ Netra Niramay Niketan, Haldia, West Bengal, India; ${ }^{27}$ Little Flower Hospital \& Research Center, Angamaly, Kerala, India; ${ }^{28}$ Ganapathy Nethralaya, Jalna, Maharashtra, India; ${ }^{29} \mathrm{HV}$ Desai Hospital, Pune, Maharashtra, India

\section{Compliance with ethical standards}

Conflict of interest The authors declare that they have no conflict of interest.

Publisher's note Springer Nature remains neutral with regard to jurisdictional claims in published maps and institutional affiliations.

\section{References}

1. World Health Organization. World health statistics 2016: monitoring health for the SDGs, sustainable development goal. Geneva: World Health Organization; 2019.

2. India State-Level Disease Burden Initiative Diabetes Collaborators. The increasing burden of diabetes and variations among the states of India: the Global Burden of Disease Study 1990-2016. Lancet Glob Health. 2018;6:e1352-62.

3. Anjana RM, Deepa M, Pradeepa R, Mahanta J, Narain K, et al. ICMR-INDIAB Collaborative Study Group. Prevalence of diabetes and prediabetes in 15 states of India: results from the ICMRINDIAB population-based cross-sectional study. Lancet Diabetes Endocrinol. 2017;5:585-96.

4. IDF Diabetes Atlas, 9th ed. 2019. https://www.diabetesatlas.org/ en/.

5. Manley SE, Hikin LJ, Round RA, Manning PW, Luzio SD, et al. Comparison of IFCC-calibrated HbA1c from laboratory and point of care testing systems. Diabetes Res Clin Pract. 2014;105:364-72.

6. Mohan V, Anbalagan VP. Expanding role of the Madras Diabetes Research Foundation-Indian Diabetes Risk Score in clinical practice. Indian J Endocrinol Metab. 2013;17:31-6.

7. Bourne RRA, Flaxman SR, Braithwaite T, Cicinelli MV, Das A, Jonas JB, Vision Loss Expert Group. et al. Magnitude, temporal trends, and projections of the global prevalence of blindness and distance and near vision impairment: a systematic review and meta-analysis. Lancet Glob Health. 2017;5: e888-97.

8. Raman R, Gella L, Srinivasan S, Sharma T. Diabetic retinopathy: an epidemic at home and around the world. Indian J Ophthalmol. 2016;64:69-75.

9. Shukla R, Gudlavalleti MV, Bandyopadhyay S, Anchala R, Gudlavalleti AS, Jotheeswaran AT, et al. Perception of care and barriers to treatment in individuals with diabetic retinopathy in India: 11-city 9-state study. Indian J Endocr Metab. 2016;20 Suppl 1:33-41.

10. Sivaprasad S. Tackling diabetic retinopathy from the grassroots. Indian J Ophthalmol. 2020;68 Suppl 1:S8-S9.

11. Scanlon PH. The English National Screening Programme for diabetic retinopathy 2003-2016. Acta Diabetol. 2017;54:515-25.

12. Diabetic retinopathy guidelines. 2012. http://www.rcophth.ac.uk. Accessed 16 Feb 2020.

13. Liew G, Michaelides M, Bunce C. A comparison of the causes of blindness certifications in England and Wales in working age adults (16-64 years), 1999-2000 with 2009-2010. BMJ Open. 2014;4:e004015.

14. Scanlon PH, Aldington SJ, Leal J, Luengo-Fernandez R, Oke J, Sivaprasad S, Gazis A, Stratton IM. Development of a costeffectiveness model for optimisation of the screening interval in diabetic retinopathy screening. Health Technol Assess. 2015;19:1-116.

15. Natarajan S, Jain A, Krishnan R, Rogye A, Sivaprasad S. Diagnostic accuracy of community-based retinopathy screening with an offline artificial intelligence system on a smartphone. JAMA Ophthalmol. 2019;137:1182-88.

16. Das T, Pappuru RR. Telemedicine in diabetic retinopathy: access to rural India. Indian J Ophthalmol. 2016;64:84-86. 